PROCEEDINGS OF THE

AMERICAN MATHEMATICAL SOCIETY

Volume 134, Number 12, December 2006, Pages 3637-3644

S 0002-9939(06)08370-5

Article electronically published on June 15, 2006

\title{
ON A CERTAIN CLASS OF LINEAR HAMILTONIAN MAPS
}

\author{
NOBUKAZU OTSUKI AND SINTARO SUZUKI \\ (Communicated by Carmen C. Chicone)
}

\begin{abstract}
We discovered a certain class of linear Hamiltonian maps which are defined by explicit time dependent Hamiltonian functions. Our method is the analogy of Moser's construction in 1986, although it is not so difficult, but we think the results are new.
\end{abstract}

\section{INTRODUCTION}

Let $(M, \omega)$ be a symplectic manifold, $\operatorname{Symp}(M, \omega)$ denote the group of symplectic maps on $(M, \omega)$ and $\operatorname{Ham}(M, \omega)$ the group of Hamiltonian maps, where Hamiltonian map is a time-1 map $\phi_{1}^{H}$ of a Hamiltonian flow $\phi_{t}^{H}$ which is defined by a time dependent Hamiltonian smooth function $H:[0,1] \times M \rightarrow \mathbb{R}$. For the details of Hamiltonian maps, see $\mathrm{AM}$, $\mathrm{HZ}$ and $\mathrm{MS}$.

If $M$ is simply connected, then $\operatorname{Ham}(M, \omega)$ coincides with the connected component $\operatorname{Symp}_{0}(M, \omega)$ of $\operatorname{Symp}(M, \omega)$. Therefore

$$
\operatorname{Ham}\left(\mathbb{R}^{2 n}, \omega_{s t}\right)=\operatorname{Symp}_{0}\left(\mathbb{R}^{2 n}, \omega_{s t}\right) .
$$

Let $S p(2 n)$ denote the group of linear symplectic maps on $\left(\mathbb{R}^{2 n}, \omega_{s t}\right)$. Since $S p(2 n)$ is connected, we have

$$
S p(2 n) \subset \operatorname{Ham}\left(\mathbb{R}^{2 n}, \omega_{s t}\right) .
$$

However, we have no general method to construct the Hamiltonian function which defines each element of $S p(2 n)$. We will treat this problem.

Let us consider the $2 n \times 2 n$ matrix $S$ with block decomposition:

$$
S=\left(\begin{array}{ll}
A & B \\
C & D
\end{array}\right) \quad A, B, C \text { and } D \text { are } n \times n \text { matrices. }
$$

Let $\mathcal{H}$ denote the set of matrices $S$ such that:

(1) $A, B, C$ and $D$ are symmetric,

(2) $A C=C A$ and $B D=D B$,

(3) $A D-C B=I$, where $I$ is a unit $n \times n$ matrix.

We will prove in $\S 3$ that each element of $\mathcal{H}$ with a condition $\operatorname{det} B \neq 0$ is a Hamiltonian map with the explicit Hamiltonian function on the symplectic manifold $\left(\mathbb{R}^{2 n}, \omega_{s t}\right)$. Moreover, in $\S 4$, we extend the set $\mathcal{H}$ in the group $\operatorname{Ham}\left(\mathbb{R}^{2 n}, \omega_{s t}\right)$. Finally we will treat the case of $2 \times 2$ symplectic matrices in $\S 5$.

Received by the editors May 17, 2004 and, in revised form, June 10, 2005.

2000 Mathematics Subject Classification. Primary 53D22, $70 \mathrm{H} 15$.

Key words and phrases. Hamiltonian map, Hamiltonian function. 


\section{Generating FUnCtions of Linear MAPS}

Let $S$ be a $2 n \times 2 n$ matrix and regard it as a linear map $\mathbb{R}^{2 n} \rightarrow \mathbb{R}^{2 n}$ sending $\left(\xi, \xi^{\prime}\right)$ to $\left(\eta, \eta^{\prime}\right)$. From now on, for $\left(\xi, \xi^{\prime}\right),\left(\eta, \eta^{\prime}\right) \in \mathbb{R}^{2 n}$, we use the following representation:

$$
S\left(\begin{array}{c}
\xi \\
\xi^{\prime}
\end{array}\right)=\left(\begin{array}{cc}
A & B \\
C & D
\end{array}\right)\left(\begin{array}{c}
\xi \\
\xi^{\prime}
\end{array}\right)=\left(\begin{array}{c}
A \xi+B \xi^{\prime} \\
C \xi+D \xi^{\prime}
\end{array}\right)=\left(\begin{array}{c}
\eta \\
\eta^{\prime}
\end{array}\right) .
$$

Definition 2.1. A smooth function $h: \mathbb{R}^{n} \times \mathbb{R}^{n} \rightarrow \mathbb{R},(\xi, \eta) \mapsto h(\xi, \eta)$ is said to be the generating function of $S$ if

$$
\xi^{\prime}=-h_{\xi}(\xi, \eta), \quad \eta^{\prime}=h_{\eta}(\xi, \eta)
$$

are satisfied globally, where $h_{\xi}$ and $h_{\eta}$ are derivatives of $h$ with respect to the variables $\xi$ and $\eta$, respectively.

Proposition 2.2. If $S=\left(\begin{array}{ll}A & B \\ C & D\end{array}\right) \in \mathcal{H}$, then $S$ is a symplectic matrix and has the generating function $h: \mathbb{R}^{n} \times \mathbb{R}^{n} \rightarrow \mathbb{R}$ with the property

$$
h_{\xi \eta}(\xi, \eta)=h_{\eta \xi}(\xi, \eta)=-B^{-1}
$$

Proof. At first, note that $S$ is a symplectic matrix if and only if

$$
A^{t} C=C^{t} A, \quad B^{t} D=D^{t} B, \quad A^{t} D-C^{t} B=I,
$$

or equivalently

$$
A B^{t}=B A^{t}, \quad C D^{t}=D C^{t}, \quad A D^{t}-B C^{t}=I .
$$

If $S \in \mathcal{H}$, then $S$ satisfies the above conditions. Consequently, in this case we have $A B=B A$ and $C D=D C$.

Next, define

$$
h(\xi, \eta)=\frac{1}{2}\left(B^{-1} A \xi \cdot \xi-2 B^{-1} \xi \cdot \eta+B^{-1} D \eta \cdot \eta\right),
$$

where $\xi \cdot \eta$ is the inner product of $\xi$ and $\eta$ in $\mathbb{R}^{n}$. Then by using the symmetric property of $A, B, C$ and $D$, we have

$$
\begin{aligned}
& h_{\xi}=\frac{\partial h}{\partial \xi}=B^{-1} A \xi-B^{-1} \eta \\
& h_{\eta}=\frac{\partial h}{\partial \eta}=-B^{-1} \xi+B^{-1} D \eta .
\end{aligned}
$$

On the other hand, since

$$
\eta=A \xi+B \xi^{\prime}, \quad \eta^{\prime}=C \xi+D \xi^{\prime},
$$

we have $\xi^{\prime}=-B^{-1} A \xi+B^{-1} \eta$, and by using the relations $A D-C B=I$ and $B D=D B$,

$$
\eta^{\prime}=C \xi+D\left(-B^{-1} A \xi+B^{-1} \eta\right)=-B^{-1} \xi+B^{-1} D \eta
$$

Thus we obtain

Obviously, we have

$$
h_{\xi}=-\xi^{\prime}, \quad h_{\eta}=\eta^{\prime} .
$$

$$
h_{\xi \eta}=\frac{\partial^{2} h}{\partial \xi \partial \eta}=-B^{-1}=\frac{\partial^{2} h}{\partial \eta \partial \xi}=h_{\eta \xi}
$$


Here we give examples of elements of $\mathcal{H}$.

Example. Let $E$ and $F$ be regular symmetric $n \times n$ matrices which satisfy $E F=$ $F E$, and $a, b, c$ and $d$ be real numbers which satisfy

$$
a d-b c=1 .
$$

Then

is in $\mathcal{H}$.

$$
S=\left(\begin{array}{ll}
a E & b F^{-1} \\
c F & d E^{-1}
\end{array}\right)
$$

\section{Linear Hamiltonian MAPS}

In this section, we prove the following theorem.

Theorem 3.1. If $S=\left(\begin{array}{ll}A & B \\ C & D\end{array}\right) \in \mathcal{H}$ and $\operatorname{det} B \neq 0$, then $S$ is a Hamiltonian map on the standard symplectic manifold $\left(\mathbb{R}^{2 n}, \omega_{\text {st }}\right)$ with the Hamiltonian function

$$
H(t, x, y)=\frac{1}{2} B^{-1}\left\{(t M+N)^{2}+M\right\} x \cdot x+(t M+N) x \cdot y+\frac{1}{2} B y \cdot y,
$$

where

$$
M=2 I-A-D, \quad N=A-I .
$$

From Proposition 2.2, $S$ has the generating function $h$ with the property $h_{\xi \eta}=$ $h_{\eta \xi}$. Therefore by using the results in Otsuki-Suzuki OS] and Suzuki [Su], the theorem is true. Here we give a direct proof for the convenience of the reader.

Proof of Theorem 3.1. For $t \in[0,1]$ and $x, p \in \mathbb{R}^{n}$, put

$$
L(t, x, p)=-\frac{1}{2} B^{-1} M x \cdot x-B^{-1}(t M+N) x \cdot p+\frac{1}{2} B^{-1} p \cdot p ;
$$

then we have

$$
L_{p}(t, x, p)=-B^{-1}(t M+N) x+B^{-1} p .
$$

From the relation

$$
y=L_{p}=-B^{-1}(t M+N) x+B^{-1} p
$$

we obtain the Legendre transformation $H(t, x, y)$ of $L(t, x, p)$ as follows:

$$
\begin{aligned}
& H(t, x, y)=p \cdot y-L(t, x, p) \\
= & ((t M+N) x+B y) \cdot y+\frac{1}{2} B^{-1} M x \cdot x \\
& +B^{-1}(t M+N) x \cdot((t M+N) x+B y) \\
& -\frac{1}{2} B^{-1}((t M+N) x+B y) \cdot((t M+N) x+B y) \\
= & \frac{1}{2} B^{-1}\left\{(t M+N)^{2}+M\right\} x \cdot x+(t M+N) x \cdot y+\frac{1}{2} B y \cdot y .
\end{aligned}
$$

Therefore the corresponding Hamiltonian system is the following:

$$
\begin{aligned}
& \dot{x}=H_{y}=(t M+N) x+B y, \\
& \dot{y}=-H_{x}=-B^{-1}\left\{(t M+N)^{2}+M\right\} x-(t M+N) y .
\end{aligned}
$$

By our method of construction in $[\mathrm{OS}]$ and $[\mathrm{Su}$, we can solve this Hamiltonian system with the initial value $x(0)=\xi$ and $y(0)=\xi^{\prime}$. In fact, put

$$
x(t)=(1-t) \xi+t \eta,
$$


and substitute this into equation (1); then we have

$$
\eta-\xi=(t M+N) x+B y .
$$

Therefore we have

$$
y=-B^{-1}(t M+N) x+B^{-1}(\eta-\xi) .
$$

By using results $\xi^{\prime}=-B^{-1} A \xi+B^{-1} \eta$ and $\eta^{\prime}=-B^{-1} \xi+B^{-1} D \eta$ in $\S 2$, we can confirm the following:

$$
\begin{aligned}
x(0) & =\xi, \quad x(1)=\eta, \\
y(0) & =-B^{-1} N x(0)+B^{-1}(\eta-\xi) \\
& =-B^{-1}(A-I) \xi+B^{-1}(\eta-\xi) \\
& =-B^{-1} A \xi+B^{-1} \eta=\xi^{\prime}, \\
y(1) & =-B^{-1}(M+N) x(1)+B^{-1}(\eta-\xi) \\
& =-B^{-1}(I-D) \eta+B^{-1}(\eta-\xi) \\
& =B^{-1} D \eta-B^{-1} \xi=\eta^{\prime} .
\end{aligned}
$$

Moreover, by differentiating equation (3) with respect to $t$, we have

$$
\begin{aligned}
\dot{y} & =-B^{-1} M x-B^{-1}(t M+N) \dot{x} \\
& =-B^{-1} M x-B^{-1}(t M+N)\{(t M+N) x+B y\} \\
& =-B^{-1}\left\{(t M+N)^{2}+M\right\} x-(t M+N) y .
\end{aligned}
$$

These equations imply that $(x(t), y(t))$ is the solution of the initial value problem whose terminal value is $(x(1), y(1))=\left(\eta, \eta^{\prime}\right)$.

Example. By this theorem, for $S=\left(\begin{array}{cc}O & -I \\ I & O\end{array}\right)$, we obtain the corresponding Hamiltonian:

$$
H(t, x, y)=-\frac{1}{2}\left\{(2 t-1)^{2}+2\right\} x \cdot x+(2 t-1) x \cdot y-\frac{1}{2} y \cdot y .
$$

The solutions of the Hamiltonian systems with initial conditions $x(0)=\xi$ and $y(0)=\xi^{\prime}$ is the following:

$$
\left\{\begin{array}{l}
x(t)=(-t+1) \xi-t \xi^{\prime} \\
y(t)=\left(-2 t^{2}+3 t\right) \xi-\left(2 t^{2}-t-1\right) \xi^{\prime} .
\end{array}\right.
$$

Solving the above equations for $\xi$ and $\xi^{\prime}$, we obtain

$$
\left\{\begin{array}{l}
\xi=-\left(2 t^{2}-t-1\right) x+t y \\
\xi^{\prime}=\left(2 t^{2}-3 t\right) x+(-t+1) y
\end{array}\right.
$$

which represents the inverse flow of the original Hamiltonian flow. We shall use these results later. 


\section{Extension of the Set $\mathcal{H}$}

Let $H, K:[0,1] \times \mathbb{R}^{2 n} \rightarrow \mathbb{R}$ be smooth Hamiltonian functions. Put

$$
\begin{aligned}
\bar{H}(t, z) & =-H\left(t, \phi_{t}^{H}(z)\right), \\
(H \# K)(t, z) & =H(t, z)+K\left(t,\left(\phi_{t}^{H}\right)^{-1}(z)\right), \\
H_{\vartheta}(t, z) & =H\left(t, \vartheta^{-1}(z)\right) \quad\left(z \in \mathbb{R}^{2 n}\right),
\end{aligned}
$$

where $\phi_{t}^{H}$ is the Hamiltonian flow corresponding to the Hamiltonian function $H$ and $\vartheta \in \operatorname{Symp}\left(\mathbb{R}^{2 n}, \omega_{s t}\right)$. The following are well known (see Hofer-Zehnder [HZ]):

$$
\begin{aligned}
\phi_{t}^{\bar{H}} & =\left(\phi_{t}^{H}\right)^{-1}, \\
\phi_{t}^{H \# K} & =\phi_{t}^{H} \circ \phi_{t}^{K}, \\
\phi_{t}^{H \vartheta} & =\vartheta \circ \phi_{t}^{H} \circ \vartheta^{-1} .
\end{aligned}
$$

Namely, $\operatorname{Ham}\left(\mathbb{R}^{2 n}, \omega_{s t}\right)$ is a group with composition as group product and admits the conjugate action by $\operatorname{Symp}\left(\mathbb{R}^{2 n}, \omega_{s t}\right)$.

Lemma 4.1. If $S \in \mathcal{H}$, then we have the following results:

(1) If $\operatorname{det} B \neq 0$, then the corresponding Hamiltonian function is

$$
\begin{aligned}
H(t, x, y)= & \frac{1}{2} B^{-1}\left\{(t M+N)^{2}+M\right\} x \cdot x \\
& +(t M+N) x \cdot y+\frac{1}{2} B y \cdot y .
\end{aligned}
$$

(2) If $\operatorname{det} C \neq 0$, then the corresponding Hamiltonian function is

$$
H(t, x, y)=H_{1 \vartheta}(t, x, y)
$$

where

$$
\begin{aligned}
H_{1}(t, x, y)= & -\frac{1}{2} C^{-1}\left\{\left(t M_{1}+N_{1}\right)^{2}+M_{1}\right\} x \cdot x \\
& +\left(t M_{1}+N_{1}\right) x \cdot y+\frac{1}{2} C y \cdot y \\
M_{1}= & M=2 I-A-D, \quad N_{1}=D-I, \quad \vartheta=\left(\begin{array}{cc}
O & -I \\
I & O
\end{array}\right) .
\end{aligned}
$$

(3) If $\operatorname{det} D \neq 0$, then the corresponding Hamiltonian function is

$$
H(t, x, y)=\left(H_{2} \# K_{2}\right)(t, x, y)
$$

where

$$
\begin{aligned}
H_{2}(t, x, y)= & -\frac{1}{2}\left\{(2 t-1)^{2}+2\right\} x \cdot x \\
& +(2 t-1) x \cdot y-\frac{1}{2} y \cdot y, \\
K_{2}(t, x, y)= & \frac{1}{2} D^{-1}\left\{\left(t M_{2}+N_{2}\right)^{2}+M_{2}\right\} x \cdot x \\
& +\left(t M_{2}+N_{2}\right) x \cdot y-\frac{1}{2} D y \cdot y, \\
M_{2}= & 2 I+B-C, \quad N_{2}=C-I .
\end{aligned}
$$

(4) If $\operatorname{det} A \neq 0$, then the corresponding Hamiltonian function is

$$
H(t, x, y)=\left(H_{3} \# K_{3}\right)(t, x, y)
$$


where

$$
\begin{aligned}
H_{3}(t, x, y)= & \frac{1}{2} A^{-1}\left\{\left(t M_{3}+N_{3}\right)^{2}+M_{3}\right\} x \cdot x \\
& +\left(t M_{3}+N_{3}\right) x \cdot y-\frac{1}{2} A y \cdot y, \\
K_{3}(t, x, y)= & -\frac{1}{2}\left\{(2 t-1)^{2}+2\right\} x \cdot x \\
& +(2 t-1) x \cdot y-\frac{1}{2} y \cdot y, \\
M_{3}= & M_{2}=2 I+B-C, \quad N_{3}=N_{2}=C-I .
\end{aligned}
$$

Proof. (1) is just the result of Theorem 3.1.

(2) is verified by

$$
\left(\begin{array}{ll}
A & B \\
C & D
\end{array}\right)=\left(\begin{array}{cc}
O & -I \\
I & O
\end{array}\right)\left(\begin{array}{cc}
D & -C \\
-B & A
\end{array}\right)\left(\begin{array}{cc}
O & -I \\
I & O
\end{array}\right)^{-1}
$$

(3) is verified by

$$
\left(\begin{array}{ll}
A & B \\
C & D
\end{array}\right)=\left(\begin{array}{cc}
O & -I \\
I & O
\end{array}\right)\left(\begin{array}{cc}
C & D \\
-A & -B
\end{array}\right)
$$

(4) is verified by

$$
\left(\begin{array}{ll}
A & B \\
C & D
\end{array}\right)=\left(\begin{array}{cc}
-B & A \\
-D & C
\end{array}\right)\left(\begin{array}{cc}
O & -I \\
I & O
\end{array}\right)
$$

Remark 4.2. If $\operatorname{det} B \neq 0$ and $\operatorname{det} C \neq 0$, then we obtain two Hamiltonian functions which define the same Hamiltonian map $S=\left(\begin{array}{ll}A & B \\ C & D\end{array}\right)$.

Set

$$
\tilde{\mathcal{H}}=\bigcup\left\{\vartheta \circ \mathcal{H} \circ \vartheta^{-1} \mid \vartheta \in \operatorname{Symp}\left(\mathbb{R}^{2 n}, \omega_{s t}\right)\right\} .
$$

Now consider the group $\mathcal{G}$ generated by $\tilde{\mathcal{H}}$ with the composition as group product; then we have the following theorem.

Theorem 4.3. Each element of $\mathcal{G}$ is a Hamiltonian map.

Note that each element of $\mathcal{G}$ can be defined by an explicit Hamiltonian function by using Lemma 4.1 and above operations.

\section{2-Dimensional CASE}

In this section, we give concrete Hamiltonian functions for all elements of $S p(2)$.

Theorem 5.1. If $S=\left(\begin{array}{ll}a & b \\ c & d\end{array}\right) \in S p(2)$, then we have the following Hamiltonian function for $S$ :

(1) If $b \neq 0$, then

$$
H(t, x, y)=\frac{1}{2 b}\{(t(2-a-d)+(a-1)) x+b y\}^{2}+\frac{1}{2 b}(2-a-d) x^{2} .
$$


(2) If $b=0$ and $c \neq 0$, then

$$
H(t, x, y)=-\frac{1}{2 c}\{c x+(t(2-a-d)+(d-1)) y\}^{2}-\frac{1}{2 c}(2-a-d) y^{2} .
$$

(3) If $b=c=0$ and $a d=1$, then

$$
\begin{aligned}
& H(t, x, y) \\
= & \frac{1}{2}\{(2 t-1) x+y\}^{2}+x^{2}-a\{(t-1)(2 t+1) x+t y\}^{2} \\
& -\frac{a}{2}\{(t(2 t-3) d-(t-1)(2 t+1)(2 t-1)) x+((t-1) d-t(2 t-1)) y\}^{2} .
\end{aligned}
$$

Proof. (1) and (2) are verified by Lemma 4.1. For (3), the process of calculus is the following. Since $S=\left(\begin{array}{cc}0 & 1 \\ -1 & 0\end{array}\right)\left(\begin{array}{cc}0 & -d \\ a & 0\end{array}\right)$, we have

$$
H(t, x, y)=H_{1} \# H_{2}(t, x, y) \text {, }
$$

where

$$
\begin{aligned}
& H_{1}(t, x, y)=\frac{1}{2}\{(2 t-1) x+y\}^{2}+x^{2}, \\
& H_{2}(t, x, y)=-\frac{a}{2}\{(2 t-1) x-d y\}^{2}-a x^{2} .
\end{aligned}
$$

The inverse flow $\left(\phi_{t}^{H_{1}}\right)^{-1}$ of $\phi_{t}^{H_{1}}$ is the following:

$$
\left(\phi_{t}^{H_{1}}\right)^{-1}(x, y)=(-(t-1)(2 t+1) x-t y,-t(2 t-3) x-(t-1) y) .
$$

Therefore

$$
\begin{aligned}
& H(t, x, y)=H_{1}(t, x, y)+H_{2}\left(t,\left(\phi_{t}^{H_{1}}\right)^{-1}(x, y)\right) \\
= & \frac{1}{2}\{(2 t-1) x+y\}^{2}+x^{2}-a\{(t-1)(2 t+1) x+t y\}^{2} \\
& -\frac{a}{2}\{(t(2 t-3) d-(t-1)(2 t+1)(2 t-1)) x+((t-1) d-t(2 t-1)) y\}^{2} .
\end{aligned}
$$

Here we give a famous example.

Example. $S=\left(\begin{array}{ll}1 & 1 \\ 1 & 2\end{array}\right)$ is the Hamiltonian map with the Hamiltonian function

$$
H(t, x, y)=\frac{1}{2} y^{2}-t x y+\frac{1}{2}\left(t^{2}-1\right) x^{2} .
$$

In fact, corresponding Hamiltonian system is

$$
\left(\begin{array}{l}
\dot{x} \\
\dot{y}
\end{array}\right)=\left(\begin{array}{cc}
-t & 1 \\
1-t^{2} & t
\end{array}\right)\left(\begin{array}{l}
x \\
y
\end{array}\right),
$$

and the solution with initial value conditions $x(0)=\xi, y(0)=\xi^{\prime}$ is

$$
x(t)=\xi+t \xi^{\prime}, \quad y(t)=t \xi+\left(t^{2}+1\right) \xi^{\prime} .
$$




\section{REFERENCES}

[AM] R. Abraham and J. Marsden, Foundations of Mechanics, Second edition. Addison-Wesley, Reading, 1978. MR 0515141 (81e:58025)

[HZ] H. Hofer and E. Zehnder, Symplectic Invariants and Hamiltonian Dynamics, Birkhauser, Basel, 1994. MR1306732 (96g:58001)

[M] J. Moser, Monotone twist mappings and the calculus of variations, Ergod. Th. \& Dynam. Sys. 6 (1986) 401-413. MR0863203 (88a:58076)

[MS] D. McDuff and D. Salamon, Introduction to Symplectic Topology, Oxford Univ. Press, 1995. MR.1373431 (97b:58062)

[OS] N. Otsuki and S. Suzuki, Monotone twist maps on $T^{*} \mathbb{T}^{n}$ and Mather's theory for minimal measures, submitted to Ergodic Theory and Dynamical Systems.

[Su] S. Suzuki, Moser's construction of time-dependent Hamiltonian function which defines a Hamiltonian map on $\mathbb{R}^{2 n}$, SUT. J. Math. 39 (2003) 105-116. MR1993843 (2005b:37115)

Department of Mathematics, Faculty of Science and Technology, Tokyo University of Science, Noda, Chiba(278-8510), Japan

E-mail address: otsuki_nobukazu@ma.noda.tus.ac.jp

Department of Mathematics, Faculty of Science and Technology, Tokyo University of Science, Noda, Chiba(278-8510), JaPan

E-mail address: suzuki_sintaro@ma.noda.tus.ac.jp 